\title{
God of the gaps: \\ a neglected reply to God's stone problem
}

\author{
Jc Beall \& A. J. Cotnoir
}

May 11, 2017

Traditional monotheism has long faced logical puzzles (omniscience, omnipotence, and more). We present a simple but plausible 'gappy' framework for addressing these puzzles. By way of illustration we focus on God's alleged stone problem. What we say about the stone problem generalizes to other familiar 'paradoxes of omni- properties', though we leave the generalization implicit. ${ }^{1}$ We assume familiarity with the proposed (subclassical) logic but an appendix is offered as a brief review.

\section{The stone problem}

The familiar problem points to a stone: ${ }^{2}$

1. Either God can create a stone which God cannot lift or God cannot create such a stone.

2. If God can create a stone which God cannot lift, then God cannot do everything.

3. If God cannot create a stone which God cannot lift, then God cannot do everything.

4. Therefore God is not omnipotent.

\footnotetext{
${ }^{1}$ Much of what we have to say extends to other 'Type-A' paradoxes in the sense of [16]: paradoxes involving the purported inconsistency of any individual omni-property. For example, our approach delivers straightforward solutions to (for example) Grim's paradox [12], Milne's paradox [15], and the like. We take no position on whether our approach can or should also be applied to either 'Type-B' paradoxes, which purport to show an inconsistency between the omni-properties, or 'Type-C' paradoxes which purport to show an inconsistency between the omni-properties and contingent features of the world.

${ }^{2}$ This presentation follows the argumentation in [14].
} 
The standard response, stemming from Aquinas [1, I.25.3], points to (3); a less standard response points to $(2)[11,18]$. We point to (1) in a theology that involves truth-value gaps in a fundamental way.

\section{God of the gaps}

On our view the stone 'problem' may in fact be seen as a theological result: namely, that logic is weaker than classical (and many other) logics, and in particular that excluded middle is not logically valid. God's essence, we suggest, invites truth-value gaps. ${ }^{3}$

\subsection{Essence against truth value}

God is a necessary being who is essentially omnipotent. On our view a key feature of God's essential omnipotence (or omni-anything) is that in some cases one can no more falsely attribute limits to God's omnipotence than one can truly do so.

God's alleged stone problem is not very different from common semantic or logical paradoxes. Somewhere along the line excluded middle is invoked on the authority of logic itself. The justification for premiss (1) of the stone argument is the same: logic. But that's a mistake in our proposed theology. Not even logic can force the limit-imposing claim

that God can create a stone that God cannot lift

or, generally,

that God can create something more powerful than God

to be false or true. On the proposed theology God is responsible for logical consequence; and there's hardly any reason - theological or otherwise - to think that God demands excluded middle of logic itself. In fact, our view is that there's good reason to think that God imposes no such constraint on logic: namely, witness 'the stone result' above!

\footnotetext{
${ }^{3}$ Strands of Christian theology talk as if there may be truth-value gaps. To our knowledge, no actual proposal on how to understand logic in such a picture has been advanced. The only explicit suggestion we are aware of is in the final two paragraphs of Dummett [10], where he suggests we need a trivalent non-intuitionist logic to account for God's timeless knowledge of truths we will come to verify.
} 


\subsection{The official response: reject (1)}

The response is to reject premiss (1) of the stone argument. Instead of being a would-be paradox for omnigod theology the stone argument delivers the result that the correct logic is a 'gappy' or 'paracomplete' consequence relation - one wherein excluded middle is not sanctioned.

Whether (2) and (3) are true or false is an independent question. By our lights there's no reason to reject these premisses; but our view is compatible

with their rejection. On our view the key theological insight is that language about 'God's limits' can result in truth-value gaps.

We fully acknowledge that there is a tome (too heavy to lift?) containing the many 'restrictivist' responses to the stone problem: responses that define omnipotence in various restricted ways. The form of argument that we highlight puts the fewest restrictions on omnipotence, and in this way might be viewed as the hardest version of the stone problem. But even the restrictivist versions may benefit from our proposed gappy theology. It may be that the corresponding restricted versions of the stone problem (e.g., 'a stone satisfying condition...') falter on the same basic point: they assume excluded middle when, as we argue, there is good reason not to do so in theology.

\subsection{Logical picture: subclassical}

Before turning to objections we need to say something about which 'gappy logic' we advance as part of the proposed theology.

An immediate question that one faces is whether, on the proposed view of logic, there are logically valid arguments that classical logic deems to be invalid. While this is a theoretical option, we reject it. As far as we can see, the problem with classical logic is that it goes too far: it classifies too many arguments as logically valid (including ones that result in excluded middle). But if classical logic finds a counterexample to an argument then we see no reason to reject such a counterexample.

With this perspective in mind we suggest a theological picture in which logic itself is properly subclassical: if an argument is logically valid then it's valid according to classical logic; but the converse fails (e.g., excluded middle).

Taking logic to be subclassical (i.e., properly weaker than classical logic) does not dictate a particular logic. This is not the place to canvass all candidates. Instead, we advance a very familiar one as part of the proposed theology. Standard thinking on logic takes logical consequence to respect 
at least familiar De Morgan behaviour among the logical expressions (viz., standard first-order expressions shy of identity). We agree with such standard thinking. A suitable candidate for logic is either Strong Kleene (K3) or First-degree Entailment (FDE). ${ }^{4}$

We note that if one accepts the truth of both (2) and (3) in the stone argument then an account of 'if' that goes beyond either K3 or FDE is required. There are plenty of candidates, and we leave debate over which is the best candidate to another venue. ${ }^{5}$

\section{Objections and Replies}

\subsection{Objection: too weak!}

There's an immediate objection to the proposed God-of-the-gaps picture. The objection stems from the fact that there are many truths of the form

$b$ can create a stone that $b$ cannot lift.

But the going account treats such claims as gappy. And this is unacceptable.

Reply. The objection rests on a mistake. Our view is not that there are no truths of the given form; there are. And from those truths it follows by $\operatorname{logic}$ - that

either $b$ can create a stone that $b$ cannot lift or $b$ cannot do so.

But our view is that logic doesn't deliver such instances of excluded middle unless, as above, something else has already delivered one of the disjuncts. In the end, whenever $p \vee \neg p$ holds for all $p$ in a theory, the explanation for its holding is not logic but rather special theory-specific constraints on the theory's consequence relation: one builds such excluded-middle behavior into the phenomenon-specific theory - a not uncommon idea in the area of subclassical logic. The theory can be very 'classical' with respect to its key predicates, even though logic itself is subclassical. ${ }^{6}$

\footnotetext{
${ }^{4} \mathrm{~K} 3$ is briefly sketched in the appendix, and both logics are laid out in many familiar places $[6,8,17]$. FDE, unlike K3, affords the logical possibility of gluts (i.e., the dual of gaps); and it is in some ways more 'balanced' than K3 [5], in that it rejects both LEM (viz., $p \therefore q \vee \neg q$ ) and EFQ (viz., $\neg q \wedge q \therefore p$ ). While gluts might be useful in a theology $[4,9,15]$, and while one of us in fact thinks that FDE is the better account of logic full stop, we herein officially advance K3 as logic in the proposed God-of-the-gaps picture. (Further debate may push for FDE.)

${ }^{5}$ See any of the many works on adding conditionals to subclassical logics $[8,17]$.

${ }^{6}$ For example, one might 'shrug' a unary predicate $P$ relative to a theory $T$ 's conse-
} 


\subsection{Objection: locating gaps}

The proposal contends that some claims that purport to limit God's power are gappy. Are any limit claims simply false? And if so which ones, and how can one tell which ones are gaps?

Reply. Some limit claims like 'God cannot create on a Wednesday' and 'God cannot violate the laws of England' are simply false. Others like 'God cannot create a stone too heavy for God to lift' are gappy. The proposal for identifying gappy limit claims is as follows:

(G) if a limit claim $L$ implies that God has limits, and the negation of $L$ implies that God has limits, then $L$ is gappy. Otherwise $L$ is false.

By 'implies' here we mean all-possible-worlds entailment. Consider the limit claim 'God cannot create on a Wednesday'. There's a world where God creates on Wednesdays, and there's no world where God is limited. So by (G) 'God cannot create on a Wednesday' isn't gappy; it's false. Now consider the limit claim 'God can do something that limits God's powers'. Any world where that's true (and there are none) is one where God's power is limited. Similarly for the target negation (viz., 'God cannot do something that limits God's powers'): any world where it's true (and there are none) is one where God's power is limited. Hence, both the claim and its negation imply that God's power is limited. This is a case of gappy limit claims. ${ }^{7}$

\subsection{Objection: whereof omnipotence?}

The project is to accommodate the traditional omnigod picture by thinking of logic as a particular subclassical - and gappy - logic. But it looks like omnipotence itself is lost if K3 (or FDE) is taken to be logic. How does one accept the omnipotence claim while rejecting some of its instances? ${ }^{8}$ If we take the line that the stone-instance of excluded middle (i.e., premiss (1))

quence relation by giving a shrug rule: $q \vdash_{T} \forall x(P x \vee \neg P x)$. (And if one were in FDE one might also do the dual for $P$ by giving a 'shriek' rule: $\exists x(P x \wedge \neg P x) \vdash_{T} q$.) See [2, 3] for discussion.

${ }^{7}$ Some theological considerations appear to suggest that some limit claims may be true. For example, one might argue that God's omnibenevolence entails that God cannot do evil. Similarly, a Christian view of the incarnation might suggest that God limited Godself in the person of Jesus. We reject that such considerations introduce genuine limits on God's power, but a full explanation requires more space than we have here.

${ }^{8}$ Of course, we are not thinking of rejection as accepting the negation. We are thinking of the proposed view as involving a rejection of both God can create too-heavy stones and its negation God cannot create too-heavy stones. The question is how to square this with an acceptance of the claim that God is omnipotent. 
should be rejected then we can't accept either disjunct. Which means that we must reject God can create a too-heavy stone... Thus, it looks like we have to reject the generalization God can do everything. And so your Godof-the-gaps theology enjoys gappiness at the price of losing omnipotence.

\subsubsection{Reply 1: omnipotence as limitless power}

In keeping with the salient spirit of much traditional doctrine, commitment to God's omnipotence is at least partly reflected in our rejecting any wouldbe limit to God's power. We reject as either false or gappy any claim that expresses a limit to God's power.

\subsubsection{Reply 2: the generalization itself}

The objection remains: what of the generalization that God can do everything? ${ }^{9}$ How, if at all, does your account accommodate this generalization?

The status of the generalization depends on the universal quantifier involved. Here, we propose a distinction between two different universal quantifiers: one is the logical one of $\mathrm{K} 3$; the other is an extra-logical one, a quantifier that goes beyond pure logical vocabulary.

Logical. The universal quantifier of logic (viz., K3) yields generalizations $\forall x \Phi(x)$ that take the lowest semantic status that the given open sentence $\Phi(x)$ takes on any assignment of objects to the variable $x .{ }^{10}$ Example: if there's no object of which the condition $\Phi(x)$ is false then $\forall x \Phi(x)$ isn't false; it is, in that case, gappy if there's some object of which $\Phi(x)$ is neither true nor false; it's true exactly if there's no object of which $\Phi(x)$ is untrue (i.e., either gappy or false). With this in mind, consider the target generalization.

If 'God can do everything' is expressed using the logical quantifier then 'God can do everything' is gappy on our account; for no claim of the form 'God can do $x$ ' is false but some such claims are gappy (witness the stone). One might think it reasonable to accept a generalization provided that it's never false $[7,13]$, in which case it might be reasonable to accept the given omnipotence generalization even though it is gappy. But if, as we are inclined to think, truth is required of acceptable claims, then the generalization of God's omnipotence (viz., 'God can do everything'), when expressed using this logical quantifier, is not acceptable. Whence the current objection.

\footnotetext{
${ }^{9}$ Throughout, we leave implicit that this generalization restricts the first-order quantifier to events, so the omnipotence claim, unpacked, becomes: 'for all $x$, if $x$ is an event then God can bring about $x$ '.

${ }^{10}$ For a precise but brief review, see appendix.
} 
Extra-logical. In subclassical logics it is common that classically equivalent characterizations fail to be equivalent in the weaker (subclassical) setting. K3 is no different. In particular, one can give semantics for two classically equivalent quantifiers that are non-equivalent in K3. What were indistinguishable quantifiers in the tightly constrained classical setting are distinct in the broader nonclassical setting. One then has a choice as to which of the two quantifiers is the appropriate one to give voice to the omnipotence generalization. We suggest that the acceptable generalization is voiced using an extra-logical quantifier - one involved in our theory of God.

Focusing on unary predicates for simplicity (and without loss of generality) the truth conditions are as follows, where $\Phi(x)$ is any context with exactly one variable $x$ free:

- $\mathcal{A} x \Phi(x)$ is true iff there's no object of which $\Phi(x)$ is false.

So long as the given predicate (or open sentence) isn't false of anything the generalization, voiced using $\mathcal{A}$, is true. This affords the truth of 'God can do everything', where the everything is the extra-logical universal quantifier. And if doctrine demands that the generalization be true in all models of our theory (all models of our theology) we can tweak things to make it so; but we leave those details to future debate. ${ }^{11}$

\section{$4 \quad$ Closing remarks}

That theology might involve truth-value gaps is an idea that may be obvious to philosophers; however, to our knowledge, an explicit account of such a theology - tied to an explicit account of logical vocabulary - has not been offered. We acknowledge that there is a lot more to say towards filling out the theological picture and its tie to the simple subclassical - in particular, gappy - account of logic. Our hope is that this paper serves as a platform for filling out a credible theology which is not a 'mere philosophical theology' but one which is in keeping with standard doctrinal accounts. By our lights,

\footnotetext{
${ }^{11}$ In the formal K3 setting the corresponding truth-in-a-model conditions, which can be subject to variable assignments (which we ignore here), are tied to the interpretation of predicates. Predicates are assigned an extension and antiextension; and a predicate is false (true) of an object iff the object is in its antiextension (extension), and gappy otherwise. The condition on the target extralogical quantifier is:

$\mathcal{A} x \Phi(x)$ is true in a model iff there's no object in the domain of the model which is in the antiextension of $\Phi(x)$.

So, $\mathcal{A} x \Phi(x)$ is true-in-a-model just if there's nothing in the domain of which $\Phi$ is false.
} 
standard doctrine does not dictate that God demands classical logic; it is compatible with nonclassical logic. The marriage of philosophically familiar nonclassical logics and philosophical theology is long overdue. ${ }^{12}$

\section{Appendix: a review of $K 3$}

We do not present K3 in full; we present only enough to make sense of the explicit proposal (and some of the objections) canvassed in the paper. Full treatments are available in textbooks on philosophical logic $[6,8,17]$.

\section{Syntax}

To simplify we restrict the syntax to monodic predicates. The syntax is exactly that of classical monadic first-order logic (without identity). The logical vocabulary is $\vee, \neg, \forall$. Constants $c_{0}, c_{1}, \ldots$ and variables $x_{0}, x_{1}, \ldots$ are the only terms. The set $\mathbb{P}$ contains (unary) predicate symbols. The standard recursive treatment defines the set of sentences.

\section{Semantics}

A K3 interpretation $I$ consists of a non-empty domain $D$, a denotation function $d$, and a variable assignment $v$, such that:

- for any constant $c, d(c) \in D$,

- for any variable $x, v(x) \in D$,

- for any predicate $P \in \mathbb{P}, d(P)=\left\langle P^{+}, P^{-}\right\rangle$, where $P^{+}, P^{-} \subseteq D$ and $P^{+} \cap P^{-}=\emptyset$.

Note well: there is no constraint on models according to which $P^{+} \cup P^{-}=D$, thereby affording 'gappy models' of a predicate - objects falling between both $P^{+}$and $P^{-}$for some $P \in \mathbb{P}^{13}$

$|\varphi|_{v}$ is the semantic value of a sentence $\varphi$ with respect to a variable assignment $v$, which is defined in the standard recursive fashion. (We leave

\footnotetext{
${ }^{12}$ Thanks to Don Baxter, Nathan Kellen, Jakob Ohlhorst, Andrew Parisi, Dave Ripley, Shawn Standefer, Andrew Tedder, and John Troyer for discussion, and also to two anonymous referees for their valuable comments.

${ }^{13}$ We note that to get FDE, which is briefly mentioned in the text, one drops the condition that $P^{+} \cap P^{-}=\emptyset$. But we say no more of FDE here. See cited textbooks for full discussion.
} 
the relevant interpretation implicit.) For atomics:

$$
|P t|_{v}=\left\{\begin{array}{l}
1 \text { if } I(t) \in P^{+} \\
0 \text { if } I(t) \in P^{-} \\
\frac{1}{2} \text { otherwise. }
\end{array}\right.
$$

The inductive clauses are as follows:

1. $|\varphi \vee \psi|_{v}=\max \left\{|\varphi|_{v},|\psi|_{v}\right\}$.

2. $|\neg \varphi|_{v}=1-|\varphi|_{v}$.

3. $|\forall x \varphi|_{v}=\min \left\{|\varphi|_{v^{\prime}}: v^{\prime}\right.$ is an $x$-variant of $\left.v\right\}$.

Conjunction, existential quantification and material implication can be defined from these in the normal way.

K3 logical consequence $\vdash_{K 3}$ is defined as preservation of value 1 . Thus, a set $\Gamma$ of sentences K3-entails sentence $\varphi$ iff no K3 interpretation assigns 1 to everything in $\Gamma$ but fails to also assign 1 to $\varphi$.

\section{Remark on excluded middle}

Let us define a logical truth to be a sentence that is K3-entailed by the empty set of premisses (equivalently, true on all K3 interpretations). We note that no instance of excluded middle (i.e., no instance of the scheme $\varphi \vee \neg \varphi)$ is logically true in K3. While this is of principal importance to the theological application of our paper the result follows from a more general result: namely, that no sentence is logically true in K3. (Let every predicate be gappy in a given interpretation. This suffices for the result.)

\section{Remark on omnipotence generalization}

Note that, as per discussion in the paper, any model in which $|\varphi|_{v}$ is gappy (i.e., $\frac{1}{2}$ ) but not false (i.e., 0) is one in which $|\forall x \varphi|_{v}$ is at best gappy. Hence, for target omnipotence generalizations using the K3 quantifier 'God can do everything' is gappy on our account. But, as per the paper, the true omnipotence generalization is expressed using the alternative quantifier.

\section{References}

[1] Thomas Aquinas. Summa Theologica. Benziger bros. edition edition, 1947. 
[2] Jc Beall. $\mathrm{LP}^{+}, \mathrm{K}^{+}, \mathrm{FDE}^{+}$and their classical collapse. Review of Symbolic Logic, 6(4):742-754, 2013.

[3] Jc Beall. A simple approach towards recapturing consistent theories in paraconsistent settings. The Review of Symbolic Logic, 6(4):755-764, 2013.

[4] Jc Beall. Christ - a contradiction: A defense of Contradictory Christology. Manuscript, 2017.

[5] Jc Beall. There is no logical negation: True, false, both, and neither. Australasian Journal of Logic, 14(1), 2017.

[6] Jc Beall and Shay Allen Logan. Logic: The Basics (2nd Edition). Routledge, Oxford, 2016.

[7] Jc Beall and David Ripley. Analetheism and dialetheism. Analysis, 64(1):30-35, 2004.

[8] Jc Beall and Bas C. van Fraassen. Possibilities and Paradox: An Introduction to Modal and Many-Valued Logic. Oxford University Press, Oxford, 2003.

[9] A. J. Cotnoir. Theism and dialetheism. Manuscript, 2016.

[10] Michael Dummett. Thought and Reality. Clarendon Press, Oxford, 2006.

[11] H. Frankfurt. The logic of omnipotence. Philosophical Review, 73(2):262-263, April 1964.

[12] P. Grim. Some neglected problems of omniscience. American Philosophical Quarterly, 20:265-276, 1983.

[13] Tim Maudlin. Truth and Paradox: Solving the Riddles. Oxford University Press, New York, 2004.

[14] G. Mavrodes. Some puzzles concerning omnipotence. Philosophical Review, 72:221-223, (1963).

[15] P. Milne. Omniscient beings are dialetheists. Analysis, 76(3):250-251, July 2007.

[16] Y. Nagasawa. A new defence of Anselmian theism. The Philosophical Quarterly, 58:577-596, 2008. 
[17] Graham Priest. An Introduction to Non-Classical Logic. Cambridge University Press, Cambridge, second edition, 2008. First edition published in 2001.

[18] R. Swinburne. The Christian God. Oxford University Press, 1994. 